\section{Mitgliederrabatt bei STATdx und RADPrimer}

Sie profitieren ab sofort als DRG-Mitglied von den Rabatten bei den Radiologie-Plattformen STATdx und RADPrimer.

\section{Kooperation zwischen DRG und Elsevier \\ $\nabla$}

Die DRG hat mit Elsevier, einem der weltweit führenden Anbieter für wissenschaftliche, technische und medizinische Informationen, Produkte und Services, einen Kooperationsvertrag vereinbart, von dem Sie als DRG-Mitglied ganz besonders profitieren:

Gemeinsam ermöglichen Elsevier und die DRG den Mitgliedern der Gesellschaft rabattierten Zugriff auf Elseviers branchenführende Radiologie-Plattformen STATdx und RADPrimer. Elsevier unterstützt damit die Ziele der DRG die medizinische Fort- und Weiterbildung von hoher Qualität zu liefern und damit die Patientenversorgung zu verbessern.

\section{Konkret: Die Rabatte bei STATdx und RADPrimer \\ $\nabla$}

Den Rabatt erhalten DRG-Mitglieder, die STATdx und RADPrimer bisher noch nicht genutzt haben; der Rabatt gilt für:

- Neue Abonnenten, die sich bis zum

16.10.2015 registrieren, und sofort ein Abonnement für 2-3 Jahre abschließen, erhalten:

> bei individuellem Abo: $25 \%$ vom Einzelhandelspreis
- bei akademischer Mitgliedschaft: $15 \%$ vom Einzelhandelspreis

- Neue Abonnenten:

1. Jahr:

- bei individuellem Abo: $20 \%$ vom Einzelhandelspreis

- bei akademischer Mitgliedschaft: 10\% vom Einzelhandelspreis

2. Jahr:

- bei individuellem Abo: 10\% vom Einzelhandelspreis
- bei akademischer Mitgliedschaft: $5 \%$ vom Einzelhandelspreis

Weitere Informationen finden Sie hier:

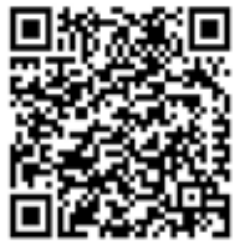

\begin{tabular}{cl}
\hline Über STATdx, & RADPrimer und Elsevier \\
STATdx & STATdx ist die weltweit größte Quelle für radiologische Inhalte. Das Produkt und des- \\
& sen Inhalte wurden von weltbekannten Radiologen aus jedem Fachgebiet verfasst und \\
& bieten umfangreiche und zuverlässige Entscheidungsunterstützung für Radiologen. \\
& Die Plattform enthält über 4000 allgemeine und komplexe Diagnosen, 200000 von Ex- \\
& perten ausgewählte und kommentierte Abbildungsbeispiele, über 1300 Differenzialdi- \\
& agnosemodule, zahlreiche Anatomieabbildungen, 20000 leicht sortierbare Patienten- \\
& fälle und grundlegende wie auch fortgeschrittene Verfahren. Es ist eine einzigartige, \\
& benutzerfreundliche, schnelle Online-Methode, um komplexe klinische Fragen zu be- \\
& antworten.
\end{tabular}

